Revue Française de Civilisation Britannique

\title{
Margaret Thatcher et les marchés financiers : le paradoxe de la déréglementation
}

Margaret Thatcher and Financial Markets: the Paradox of Deregulation

\section{Marie-Claude Esposito}

\section{CpenEdition}

\section{Journals}

Édition électronique

URL : http://journals.openedition.org/rfcb/4805

DOI : $10.4000 /$ rfcb.4805

ISSN : 2429-4373

Éditeur

CRECIB - Centre de recherche et d'études en civilisation britannique

Référence électronique

Marie-Claude Esposito, « Margaret Thatcher et les marchés financiers : le paradoxe de la

déréglementation », Revue Française de Civilisation Britannique [En ligne], XXIV-4 | 2019, mis en ligne le 25 novembre 2019, consulté le 10 décembre 2020. URL : http://journals.openedition.org/rfcb/4805 ; DOI : https://doi.org/10.4000/rfcb.4805

Ce document a été généré automatiquement le 10 décembre 2020.

Revue française de civilisation britannique est mis à disposition selon les termes de la licence Creative Commons Attribution - Pas d'Utilisation Commerciale - Pas de Modification 4.0 International. 


\title{
Margaret Thatcher et les marchés financiers : le paradoxe de la déréglementation
}

\author{
Margaret Thatcher and Financial Markets: the Paradox of Deregulation
}

\author{
Marie-Claude Esposito
}

1 Quand Margaret Thatcher arriva au pouvoir en mai 1979, le Royaume-Uni était à la dérive. La dégradation de l'économie, progressive au cours des années 1960, s'était accélérée au cours de la décennie suivante : la croissance y était inférieure à celle de ses voisins européens, notamment la France et l'Allemagne, son taux annuel ne dépassant pas $1,5 \%$ entre 1973 et 1979 , si bien que sa part dans la production industrielle mondiale avait régressé de $20,5 \%$ à $9 \%$ entre 1955 et 1976 ; le chômage était en augmentation constante, et son taux atteignait $5,5 \%$ de la population active, pendant que le taux d'inflation atteignait les $10,5 \%$ en moyenne annuelle. Le Royaume-Uni était devenu l'homme malade de l'Europe. En plus de connaître un important déclassement par rapport aux autres pays membres de l'OCDE, il avait dû en 1976, pour restaurer la confiance dans la livre sterling, solliciter un prêt de 3,6 milliards de dollars auprès du Fonds Monétaire International. Enfin, l'action du gouvernement travailliste de James Callaghan s'était trouvée totalement paralysée au cours de l'hiver du mécontentement (winter of discontent 1978-1979) par la multiplication des conflits sociaux.

2 Pour le nouveau Premier ministre et son équipe, une thérapie de choc était nécessaire pour redresser l'économie britannique. La politique économique des trois mandats de Margaret Thatcher entre 1979 et 1990 s'est donc voulue en rupture totale avec les politiques macro-économiques keynésiennes mises en œuvre depuis la fin de la Seconde Guerre mondiale, tant par les gouvernements conservateurs que travaillistes, politiques qui n'avaient pas permis une croissance durable de l'économie mais avaient abouti à créer à la fois inflation et chômage ${ }^{1}$. Elle comporta deux grands volets. Le premier avait pour objectif la lutte contre l'inflation, l'utilisation des instruments monétaires et budgétaires, grâce à la mise en place d'une Stratégie financière à moyen terme (SFMT) devant créer un cadre macro-économique stable et non inflationniste ; le 
second visait à réduire le rôle de l'État dans l'économie et à améliorer les performances de l'offre globale, dont dépendait la croissance, ce qui impliquait d'une part de déréglementer, c'est-à-dire de supprimer tous les obstacles qui, sur les différents marchés (capital, travail, produits), empêchaient les mécanismes de marché de jouer leur rôle, et d'autre part de privatiser des pans entiers du secteur public.

Cet article se limitera à l'étude de la déréglementation des marchés financiers, dont la contribution en termes de production, d'emploi et d'exportations de services augmentait depuis le début des années 1970. Grâce à sa situation géographique entre Tokyo et New York, la place financière de Londres jouissait d'un avantage comparatif indéniable au moment où se mettait en place un système financier intégré et global, le développement des nouvelles technologies de l'information rendant possible le fonctionnement des marchés financiers en continu 24 heures sur 24 . Toutefois, devant les scandales qui éclatèrent dans la City, le gouvernement mit en place, au cours de la même période, un système de surveillance statutaire des acteurs et des marchés financiers, si bien que l'économiste Philip Booth a pu intituler un de ses articles "Thatcher: le mythe de la déréglementation $»^{2}$. Après avoir repéré les forces et les faiblesses de la City à la fin des années 1970 (partie 1), cet article présentera les principales mesures de déréglementation prises dans la sphère financière durant les deux premières mandatures de Margaret Thatcher pour provoquer l'adaptation structurelle de ce secteur, ainsi que leurs conséquences (partie 2), avant d'analyser pourquoi et comment le gouvernement en vint à élargir le champ de la réglementation financière et à durcir la réglementation bancaire (partie 3).

\section{La City des années 1970}

4 Si la City ne jouissait plus du statut de première place financière du monde à l'issue de la Seconde Guerre mondiale, New York l'ayant détrônée bien que le dollar n'eût pas encore acquis le statut de première devise internationale, force est de constater que sa partie internationale parvint peu à peu, au cours des années 1960 et 1970, à reconquérir le terrain perdu, à partir du moment où les grandes banques commerciales se mirent à utiliser le dollar et non plus le sterling pour financer leurs activités internationales ${ }^{3}$. En effet, depuis l'innovation de la Midland Bank en 1954 - une opération d'arbitrage sur taux d'intérêt pour attirer les dépôts en dollars dans la City (les eurodollars) qui étaient ensuite convertis en livres sterling sur le marché à terme ${ }^{5}-$, certaines banques s'étaient mises à utiliser le dollar, lorsqu'il leur fut interdit, au moment de l'affaire de Suez de 1956, de financer des crédits en sterling pour des opérations en dehors de la zone sterling. L'ingéniosité de la Midland Bank venait de conduire les banques de la City à abandonner la livre sterling au profit du dollar, comme monnaie de transaction dans leurs activités à l'international.

Bien que l'utilisation des dollars par les banques de la City contournât la réglementation des changes imposée par le gouvernement, la Banque d'Angleterre (Bank of England, $\mathrm{BoE})$, responsable de la surveillance des banques, laissa faire. Cette innovation, en effet, limitait les effets négatifs, temporairement du moins, provoqués par les flux entrants de dollars sur la balance britannique des paiements, dont l'équilibre, depuis la fin de la Seconde Guerre mondiale, n'était pas considéré, par les gouvernements successifs, comme une contrainte imposée à la politique économique mais comme un objectif en soi. Ce faisant, la BoE facilita la diffusion de l'innovation auprès des institutions 
financières qui intervenaient dans la partie internationale de la City. Très rapidement, les banques étrangères installées à Londres (surtout américaines et japonaises), dont le nombre doubla quasiment au cours des années $1960^{6}$, les banques d'outre-mer (overseas banks), et les banques d'affaires britanniques (merchant banks) dominèrent ce marché off-shore des eurodollars, dont le développement n'était entravé par aucune réglementation. Alimenté par les dépôts des banques et des grandes multinationales américaines, par ceux de la Banque des Règlements internationaux (BRI), et ceux des banques centrales des pays de la Communauté Économique Européenne (CEE) ${ }^{7}$, ce marché allait redonner à la City le rôle international de tout premier plan qu'elle avait perdu à l'issue du second conflit mondial, et ce en dépit du déclin du sterling comme monnaie internationale. Puis, les deux chocs pétroliers $(1973,1978)$ contribuèrent à la croissance exponentielle de ce marché, les pays producteurs de pétrole souhaitant détenir leurs avoirs sous forme de dépôts bancaires, qui étaient ensuite recyclés par les banques étrangères de la City grâce à une autre innovation des années 1960 : celle des crédits syndiqués. Également appelés eurocrédits, ces prêts à moyen terme et à taux variable, financés à partir des eurodollars, étaient accordés par des consortiums bancaires, car le montant des opérations était si élevé qu'aucune banque ne pouvait, seule, en supporter le risque. Sur 17 syndicats créés entre 1964 et 1971, la City en accueillait treize, Paris trois et Bruxelles un; trois merchant banks britanniques (Warburg, Rothschild et Hambro) étaient chefs de file des vingt premiers consortia, les banques étrangères dominant également le marché dans ce domaine ${ }^{8}$. En 1979, on dénombrait 30 syndicats à Londres.

6 À côté de la partie internationale de la City, il existait une autre City qui était, elle, centrée sur le financement de l'économie nationale. La liste des institutions financières gravitant dans cette City s'était allongée au cours des années 1950 et 1960, et leurs activités étaient devenues plus nombreuses. Pour répondre aux nouveaux besoins des agents économiques, des sociétés de prêts immobiliers (building societies), des maisons de financement des ventes à tempérament, des sociétés de gestion de portefeuilles, des sociétés de leasing et de factoring côtoyaient désormais les banques commerciales (clearing banks), qui s'étaient mises à diversifier leurs activités, et les maisons d'escompte (discount houses) qui souscrivaient chaque semaine la totalité des nouvelles émissions de bons du Trésor.

7 L'apparition de ces nouveaux acteurs avait entraîné une reconfiguration du marché de l'escompte qui, au sortir de la guerre et jusqu'au milieu des années 1960, assurait le refinancement de l'ensemble des banques grâce au rôle des maisons d'escompte. Intermédiaires entre la Banque d'Angleterre et les autres banques, ces dernières acceptaient des prêts au jour le jour et à court terme, et prenaient des effets en pension, ce qui permettait la régulation de la liquidité pour l'ensemble du système bancaire. De nouveaux marchés en sterling, dits marchés parallèles ou marchés secondaires, avaient vu le jour à côté du marché de l'escompte : marché des prêts aux collectivités locales pour financer la reconstruction du pays après la guerre, marché des ventes à tempérament pour accompagner l'essor de la consommation de masse, marché interbancaire, et marché des certificats de dépôts. Puis, ils étaient devenus des compartiments du marché de l'escompte, grâce à l'activité des banques commerciales qui intervenaient sur tous ces marchés.

8 La City des années soixante-dix était donc bicéphale, la City internationale et la City "domestique» étant séparées par le mur invisible du contrôle des changes. La 
première, centrée sur les euromarchés, prospérait en échappant à toute réglementation. Elle regroupait les banques étrangères américaines, japonaises et européennes, qui avaient organisé le retrait de la livre sterling et travaillaient en dollar uniquement avec les agents non-résidents. Il s'ensuit que les problèmes posés par la livre sterling en tant que monnaie de réserve ne les concernaient plus. Du reste la Banque d'Angleterre et le Treasury n'y trouvaient rien à redire, car ils souhaitaient que la City redevienne un grand centre financier international ${ }^{10}$, le Treasury appréciant de surcroît les rentrées fiscales et les créations d'emploi venant des banques étrangères ${ }^{11}$.

La City " domestique ", en revanche, était celle des banques commerciales britanniques, des sociétés de prêts immobiliers, des compagnies d'assurance et de la Bourse de Londres (le London Stock Exchange, LSE). Toutes ces institutions, qui travaillaient en livre sterling avec les agents résidents, étaient protégées par des barrières institutionnelles, comme l'accord de cartel tripartite entre les banques commerciales, les maisons d'escompte et la $\mathrm{BoE}^{12}$, et bénéficiaient de pratiques restrictives qui limitaient la concurrence et l'entrée de nouveaux acteurs sur les différents marchés. L'autorégulation était de mise, même si, pour prévenir la dépréciation du sterling, les banques devaient respecter certains ratios imposés par la Banque d'Angleterre, et si le niveau des taux d'intérêt était décidé par le Treasury dans le cadre de la politique monétaire.

10 La City internationale et la City « domestique " allaient-elles pouvoir poursuivre leur cohabitation avec les bouleversements qu'allait connaître le système monétaire international à partir du milieu des années 1970 et l'accélération de l'intégration du marché financier mondial?

\section{La déréglementation financière}

11 Entre 1979 et 1989, le gouvernement britannique prit pas moins de dix mesures de déréglementation des marchés financiers. Il s'attaqua d'abord au contrôle des changes, dont le démantèlement, par étape, durant les six premiers mois de la législature, allait rendre toute mesure d'encadrement du crédit inefficace, et battre en brèche le caractère restrictif de sa politique monétaire. L'analyse se limite aux mesures dont les effets se développèrent en cascade.

\section{La suppression du contrôle des changes}

12 Introduit pour une période temporaire au début de la Seconde Guerre mondiale, le contrôle des changes, qui avait été prolongé par le Control Exchange Act de 1947, était toujours en vigueur quand Margaret Thatcher arriva au pouvoir en mai 1979. Bien qu'en partie responsable de la perte de compétitivité de la City au cours des deux décennies qui suivirent la guerre, il bénéficiait d'un certain consensus auprès des élites politiques et financières. Seule la BoE, chargée de sa mise en œuvre, et quelques fonctionnaires du Treasury souhaitaient vraiment sa disparition ${ }^{13}$. D'après Geoffrey Howe, premier chancelier de l'Échiquier de Margaret Thatcher qui, en novembre 1978, avait soulevé la question de son bien-fondé lors du premier débat parlementaire sur le système monétaire européen (SME), il fallut attendre une publication de février $1979 \mathrm{du}$ très libéral Institute for Economic Affairs (IEA) pour que la question de sa suppression soit abordée publiquement ${ }^{14}$. 

mentionnée dans les deux documents annonçant la stratégie économique du parti conservateur The Right Approach to the Economy ${ }^{15}$ de 1977 et le Manifeste électoral du Parti conservateur pour les élections législatives de $1979^{16}$. Nigel Lawson, qui occupait à l'époque le poste de Financial Secretary ${ }^{17}$, l'évoqua toutefois durant la campagne électorale dans les colonnes du Financial Weekly qui parut le 20 avril $1979^{18}$.

Cette mesure, qui allait avoir des conséquences très importantes pour la City et pour la mise en œuvre de la politique monétaire, avait été recommandée à Margaret Thatcher, dès le 4 mai 1979, par un ami proche (l'économiste Douglas Hague) qui, pour tenter d'enrayer l'augmentation $d u$ taux de change préjudiciable pour les entreprises britanniques, préconisait, à la différence de la BoE et du Treasury, de laisser jouer les forces du marché pour contrecarrer l'augmentation de l'excédent de la balance des capitaux $^{19}$. Ce même jour, le chancelier de l'Échiquier avait reçu un courrier du Gouverneur de la $\mathrm{BoE}^{20}$, accompagné d'une note technique sur la politique de change à mener pour tenter de ralentir l'appréciation du taux de change due essentiellement à l'augmentation du prix du pétrole de la mer du Nord. Si celle-ci contribuait à lutter contre l'inflation, elle était préjudiciable aux entreprises britanniques, en conséquence de quoi, la BoE recommandait d'assouplir le contrôle des changes.

C'est bien la volonté de rendre l'économie plus compétitive, ce qui impliquait de libéraliser les marchés, et non pas l'idée selon laquelle la déréglementation serait bénéfique pour la place financière de Londres, qui prévalut pour décider de supprimer le contrôle des changes. C'est du moins ce que l'on peut déduire d'un courrier envoyé par le chancelier de l'Échiquier Howe à Margaret Thatcher le 11 octobre 1979, courrier qui détaillait les mesures que le Treasury comptait prendre pour « (...) poursuivre notre objectif de libéraliser l'économie $»^{21}$. La BoE l'avait informé des conséquences probables de la suppression du contrôle des changes ${ }^{22}$, à savoir que les investisseurs britanniques se tournent davantage vers l'étranger, et que les entreprises britanniques aient des difficultés à trouver du capital.

Si Lawson penchait pour une suppression rapide du contrôle des changes, Howe était, lui, en faveur d'une suppression par étape. Quant à Margaret Thatcher, elle était hésitante et il fallut la convaincre du caractère indispensable de cette mesure ${ }^{23}$, l'afflux de capitaux, provoqué par l'appréciation du sterling en raison de son statut de pétrodevise, pesant sur la compétitivité des entreprises britanniques. En libéralisant les mouvements de capitaux, le gouvernement pouvait espérer, à court terme, que des sorties de capitaux viendraient compenser, au moins en partie, les entrées de capitaux, ce qui pousserait le taux de change à la baisse et, à plus long terme, que les sorties de capitaux, source de revenus, contribueraient à l'équilibre de la balance des paiements. Mais, comme le souligne Lawson, il était impossible de prévoir quelle serait l'ampleur des fuites de capitaux provoquée par la suppression du contrôle des changes ${ }^{24}$, et personne à la BoE ou au Treasury n'envisagea les conséquences que cette mesure allait avoir sur la politique monétaire britannique ${ }^{25}$.

17 Un premier train de mesures ${ }^{26}$ fut annoncé par le chancelier Howe dès le 12 juin 1979 au cours de la déclaration budgétaire ${ }^{27}$. Il devint désormais possible d'obtenir des devises, au cours officiel, pour effectuer des investissements à l'étranger, le montant maximal autorisé étant de 5 millions de livres par projet. De plus, l'obligation de rapatrier deux tiers des bénéfices obtenus à l'étranger fut supprimée, et les allocations personnelles accordées pour les voyages et l'achat de biens à l'étranger furent 
augmentées. Enfin, l'obligation de couvrir à $115 \%$ les investissements de portefeuille par des emprunts en devises étrangères fut supprimée, de même que le contrôle sur les importations d'or et de pièces de monnaie.

Le mois suivant, le 19 juillet, Howe décida, sur les recommandations de Lawson ${ }^{28}$, de libéraliser davantage les investissements de portefeuille effectués à l'intérieur de la CEE. Les résidents du Royaume-Uni obtinrent le droit d'investir dans des titres libellés dans une monnaie de la CEE ou émis par une organisation internationale, telle que la Banque mondiale.

Vers le 19 septembre, Lawson et des hauts fonctionnaires du Treasury recommandèrent à Howe d'en finir avec les restrictions qui subsistaient en matière de change, pendant que le conseiller économique en chef du Treasury, Fred Atkinson, le mettait en garde contre les risques encourus. Howe rappelle qu'il fut nécessaire de revenir plusieurs fois à la charge auprès du Premier ministre pour obtenir son accord, et que ce n'est qu'à l'issue d'une ultime réunion avec Howe, le gouverneur de la BoE, Nigel Lawson, Peter Carrington et John Nott que Margaret Thatcher finit par se laisser convaincre ${ }^{29}$.

La suppression des dernières restrictions en matière de change, à l'exception de celles concernant la Rhodésie, fut annoncée à la Chambre des Communes le 23 octobre, le Cabinet n'étant prévenu que quelques heures auparavant, ce qui mécontenta fortement le ministre de l'Environnement Michael Heseltine. Elle entraîna dans son sillage la fermeture d'un département entier de la BoE, qui comptait 750 personnes, et le redéploiement de 25 personnes au sein du Treasury ${ }^{30}$.

21 Très rapidement, les investisseurs institutionnels se mirent à accumuler des actifs à l'étranger; mais la quasi-totalité des opérations échappait aux institutions britanniques (merchant banks et sociétés de bourse) et ne profitait qu'aux banques étrangères, c'est-à-dire à la City internationale et non à la City domestique. Si cet effet avait été largement sous-estimé par les autorités monétaires (Treasury et BoE) ${ }^{31}$, le gouvernement allait ensuite reprendre l'argument de la Bourse de Londres et plus généralement de la City, selon lequel la suppression du contrôle des changes créerait les conditions pour que Londres consolide sa position comme centre financier international de première importance ${ }^{32}$.

\section{La libéralisation des marchés financiers et le Big Bang}

Ce processus avait en fait commencé dès septembre 1971 avec la mise en place de la réforme "Concurrence et contrôle du crédit " (Competition and Credit Control). Celle-ci avait autorisé les banques commerciales à intervenir sur le marché des euro-monnaies, créé les conditions d'une meilleure concurrence entre les banques et les institutions financières non bancaires, et modifié le contrôle du crédit en redonnant aux mécanismes de marché plus d'importance, c'est-à-dire en substituant aux mesures de contrôle direct une politique de taux et d'open market ${ }^{33}$. Mais deux ans après cette réforme d'inspiration très libérale, la crise bancaire de 1973-1974, qui avait débuté comme une crise de spéculation immobilière pour évoluer rapidement en une crise de spéculation sur les changes, avait contraint la $\mathrm{BoE}$ à surveiller les banques d'une manière un peu plus formelle. Cela l'avait conduite à créer un département chargé de la supervision bancaire, et à soumettre à nouveau l'augmentation des dépôts des banques commerciales à un contrôle quantitatif, en introduisant le système des dépôts spéciaux supplémentaires (Special Supplementary Deposits), plus connu sous le nom de corset. 
Proche du système français des réserves obligatoires, il obligeait les banques commerciales à geler auprès de la BoE un montant croissant de leurs dépôts, lorsque leur rythme d'augmentation dépassait un certain taux.

\section{Rendre le système financier plus concurrentiel} institutions, qui se livrèrent une concurrence acharnée pour gagner des parts de marché. Elles accordèrent des facilités de prêts aux ménages qui choisirent de s'endetter auprès des banques plutôt qu'auprès des sociétés de prêts immobiliers pour acheter des actifs réels. D'où une forte augmentation de l'endettement des ménages auprès du système bancaire et une forte diminution du taux d'épargne nette des ménages. Par ailleurs, les banques se mirent à offrir des produits d'épargne rémunérés par un intérêt en rapport avec celui du marché, ce qui entraîna une ré-allocation de l'épargne liquide au profit des banques et au détriment des building societies, car il était plus intéressant pour les épargnants de détenir des actifs financiers offerts par les banques. Ces mouvements de ré-allocation de portefeuille entrèrent en contradiction avec les objectifs de la politique monétaire : en faisant revenir dans le circuit bancaire des actifs qui l'avaient quitté, ils provoquaient un gonflement de la masse monétaire, au moment où le gouvernement s'efforçait de réduire son rythme de croissance.

\section{La loi sur les sociétés de prêts immobiliers (Building Societies Act,} 1986)

En octobre 1983, le cartel qui protégeait ces institutions s'effondra en raison de la concurrence des banques commerciales qui venaient d'investir le marché des prêts immobiliers, et de l'arrivée d'institutions spécialisées dans les prêts hypothécaires surtout américaines.

Pour rendre le système financier plus concurrentiel, le gouvernement fit voter le Building Societies Act, 1986, pour que ces établissements spécialisés dans le financement $\mathrm{du}$ logement puissent offrir à leurs clients d'autres produits financiers comme, par exemple, l'ouverture et la gestion de comptes de dépôts, l'achat et la vente de titres financiers et le courtage d'assurance. La loi donnait également aux sociétés de prêts immobiliers le droit d'abandonner leur statut mutualiste et de devenir des sociétés par actions, ce qui leur permettait d'être cotées à la Bourse de Londres et d'émettre des actions. Grâce à ces mesures de libéralisation, qui contribuaient à supprimer les frontières traditionnelles entre les différents intermédiaires financiers, le 
gouvernement espérait une diminution des coûts de l'intermédiation financière sous les effets de la concurrence. Une nouvelle autorité de régulation fut créée, la Building Societies Commission (voir infra).

\section{La réforme du marché boursier : le Big Bang de 1986}

27 En dépit de son nom, la réforme du LSE n'est pas arrivée d'un seul coup. On peut faire remonter le processus conduisant au Big Bang à la suppression du contrôle des changes, dont un des premiers effets fut d'exposer la Bourse de Londres à la concurrence internationale, alors que, jouissant d'un monopole pour les transactions sur les actions britanniques et les fonds d'État, elle était sans doute le marché financier de la City le plus protégé. "Exemple achevé du particularisme britannique $~^{34}$, c'était un club très fermé dont les règles de fonctionnement remontaient à sa création en 1812. Celles-ci n'autorisaient pas les banques britanniques et les courtiers étrangers à mener une activité sur le marché boursier.

Lorsque les Conservateurs arrivèrent au pouvoir, le LSE faisait l'objet d'une plainte. Déposée en 1978, devant le Tribunal des pratiques restrictives, par le Bureau de la concurrence loyale (Office of Fair Trading), elle avait pour objet de savoir si deux particularités du LSE - les commissions fixes et l'interdiction faites aux non-résidents et aux banques d'intervenir sur le marché boursier - constituaient des pratiques restrictives. Le gouvernement conservateur décida, dans un premier temps, de laisser la procédure suivre son cours ; mais il revint sur cette décision, lorsqu'il apparut que le LSE allait être condamné, ce qui risquait de rendre le marché boursier très instable. Finalement, et à la suite de longues tractations ${ }^{35}$ entre le nouveau ministre du Commerce et de l'Industrie, Cecil Parkinson, et le président du LSE, Gordon Richardson, un compromis (le Goodison-Parkinson Agreement), fut trouvé : le gouvernement retirait sa plainte dès lors que le LSE acceptait de se réformer.

La réforme mise en place le 27 octobre 1986 supprima la capacité unique ${ }^{36}$, les commissions fixes et les barrières entre le marché des fonds d'État et les marchés d'actions et autres titres financiers; elle ouvrit le LSE aux banques britanniques et étrangères, et le gouvernement décida de réduire le droit de timbre, pour que le marché boursier londonien puisse lutter à armes égales avec Wall Street et Tokyo. Enfin, la Bourse de Londres se dota d'un système ${ }^{37}$ permettant la cotation en continu 24 heures sur 24 de 3500 titres nationaux et internationaux, et la participation de tous les agents à la cotation; celui-ci fut complété en février 1987 par un système de transactions électroniques : le TOPIC ${ }^{38}$. La salle du rez-de-chaussée, le floor, où s'étaient effectuées les transactions depuis l'origine, fut fermée et remplacée par une immense salle des marchés au premier étage abritant des centaines d'ordinateurs.

Immédiatement après le Big Bang, les banques commerciales qui avaient été écartées de la Bourse de Londres, et qui étaient maintenant concurrencées par les sociétés d'investissement immobilier, se lancèrent dans une stratégie de banque d'investissement (investment banking) ; mais, devant les pertes accumulées au début des années 1990, elles durent réduire la voilure et revenir à l'activité de banque de détail (retail banking). Pour les banques d'affaires, qui rachetèrent les maisons de courtage pour augmenter leur potentiel à l'international, le choc fut encore plus rude et, après le choc boursier de 1987, elles furent balayées par les banques d'investissement 
américaines et les grandes banques universelles européennes. Ainsi, sous les effets de la concurrence, la place financière de Londres amorçait une nouvelle mutation ${ }^{39}$.

\section{L'évolution de la réglementation financière}

31 Si la déréglementation financière cherche à améliorer l'efficience du système en introduisant davantage de concurrence, ce qui, en diminuant les coûts et en améliorant la qualité des produits, doit normalement bénéficier aux consommateurs, elle peut également conduire à des effets négatifs prenant la forme de prises de risques excessifs qui menacent la stabilité du système et sont préjudiciables aux déposants. C'est pourquoi il est nécessaire de mettre en place une réglementation prudentielle adéquate des acteurs financiers.

Devant l'évolution des marchés financiers de la City, le gouvernement conservateur comprit très vite que la réglementation en vigueur était inadaptée, et qu'il était nécessaire d'améliorer la surveillance des activités financières pour renforcer la protection des investisseurs. Trois lois furent votées dans la foulée du Big Bang qui modifièrent radicalement la surveillance des acteurs et des marchés financiers.

\section{La loi sur les services financiers de 1986 (Financial Services Act,} 1986)

33 Le projet de loi fut l'objet de plusieurs débats avant le vote de la loi. La nouvelle législation confia les compétences de surveillance au ministère du Commerce et de l'Industrie (DTI) qui les transféra au Securities and Investment Board (SIB), organisme de droit privé financé par des fonds privés, et dont le président était nommé conjointement par le DTI et la BoE. Elle organisa l'auto-réglementation du système financier dans un cadre légalement défini, en mettant en place la surveillance des fonctions et non plus celle des institutions financières. Le SIB fut doté de compétences très importantes, puisqu'il était responsable de l'agrément et de la surveillance des organismes d'autorégulation (Self-Regulating Organizations, SRO), des bourses (Recognized Investment Exchanges, RIE) et des organisations professionnelles (Recognized Professional Bodies, RPB), tels que les cabinets d'experts comptables, tous ces acteurs devant préparer un code de bonne conduite. Seuls la Banque d'Angleterre, les compagnies d'assurance et les organismes mutualistes échappaient au contrôle du SIB. La loi exigeait des opérateurs, après obtention de l'agrément, de signer avec chaque client un accord écrit fixant les conditions d'achat ou de vente, et de donner au client et à l'autorité de régulation une information régulière sur l'évolution de la transaction.

Ce système, très complexe, fut rapidement l'objet de critiques, les uns l'accusant de n'être qu'une bureaucratie inutile, et les autres de ne pas échapper à la « capture du régulateur » (regulatory capture ${ }^{40}$ ), situation qui avait toute chance d'aboutir à une totale inefficacité du système de surveillance. Soulignons toutefois que Lawson, dans ses mémoires, rend les professionnels du secteur en partie responsables du caractère bureaucratique de la législation, car ils ne firent pas bon usage de la longue période de consultation précédant le vote de la législation ${ }^{41}$. 


\section{La loi sur les sociétés de prêts immobiliers (Building Societies Act,} 1986)

Votée en 1986, ses principales dispositions n'entrèrent en vigueur que le $1^{\mathrm{er}}$ janvier 1987. Cette loi, qui avait autorisé les sociétés de prêts immobiliers à mener les mêmes activités que les banques, confia la surveillance de ces institutions, devenues les concurrentes des banques commerciales, à un nouvel organisme, la Building Societies Commission, qui se substitua au Registrar of Friendly Societies. Si les sociétés de prêts immobiliers pouvaient désormais obtenir des fonds sur les marchés de gros (wholesale markets), la loi leur imposait toutefois que $80 \%$ de leurs avoirs proviennent de fonds de détail. Leurs actifs étaient maintenant divisés en trois catégories: les prêts hypothécaires, les créances garanties, et les créances non garanties; mais les montants des deux dernières catégories devaient rester très faibles tant que ces institutions conservaient leur statut mutualiste.

\section{La loi bancaire de 1987 (Banking Act, 1987)}

En 1984, soit dix ans après la crise bancaire de 1974, la City fut secouée par une crise qui allait révéler des défaillances dans la surveillance des établissements de crédit. Celle-ci durait depuis une semaine quand, le $1^{\text {er }}$ octobre, le chancelier de l'Échiquier Nigel Lawson, qui se trouvait à Washington pour les réunions annuelles du FMI et de la Banque Mondiale, en fut informé. La banque Johnson Matthey Bankers (JMB) était sur le point de faire faillite et risquait d'entraîner dans son sillage la maison de courtage Johnson Matthey, spécialisée dans les transactions sur l'or, et une filiale de la Midland Bank: Samuel Montagu. Le chancelier n'eut que quelques minutes pour décider s'il devait accorder la garantie du Treasury au plan de sauvetage organisé par le BoE, ce qu'il refusa de faire tout en donnant néanmoins le feu vert à la banque centrale pour l'organisation du sauvetage par les grandes banques commerciales et plusieurs banques d'affaires ${ }^{42}$.

Le 17 décembre 1984, il informa le Parlement de la mise en place d'une commission chargée d'évaluer le système de surveillance des banques et de faire des recommandations. Placée sous la présidence du Gouverneur de la BoE, Robin Leigh Pemberton, elle comptait parmi ses membres une équipe du Treasury dirigée par le Secrétaire Permanent, Peter Middleton, et un représentant des banques commerciales en la personne de Deryk Vander-Weyer, qui avait été vice-président de la Barclays. Le rapport de cette commission, publié le 20 juin 1985, montra que le Département de la Banque d'Angleterre chargé de la surveillance des banques avait failli dans sa mission, et recommanda une réforme complète du système de surveillance, la loi bancaire de 1979 n'étant plus adaptée à la City telle qu'elle était devenue.

La loi bancaire de 1987 supprima la distinction introduite par celle de 1979 entre les banques reconnues (recognized banks), seules habilitées à utiliser le mot banque dans leur raison sociale, et les institutions autorisées à recevoir des dépôts (licensed-deposit takers), qui jouissaient de critères moins stricts quant au niveau d'actifs financiers nécessaires pour garantir leurs dépôts. Pour assurer une couverture suffisante du risque, la nouvelle loi introduisit des règles qui étaient applicables à tous les établissements de crédit. Elle imposa aux auditeurs de transmettre aux autorités de surveillance les informations paraissant nécessaires. Enfin, et malgré l'opposition de la 
Banque d'Angleterre, qui ne s'y résolut qu'après que Lawson lui eût fait comprendre que Margaret Thatcher était prête à lui retirer la surveillance des banques pour la confier à une agence indépendante ${ }^{43}$, elle créa un Comité de la surveillance bancaire (Board of Banking Supervision) présidé par le Gouverneur de la BoE, qui comptait deux tiers de membres non banquiers, et qui avait une mission de conseil auprès de la Banque centrale. Celle-ci pouvait rejeter ses conseils, mais était tenue d'en informer le chancelier de l'Échiquier. Lawson avait préféré ne pas retirer la surveillance des banques à la Banque d'Angleterre, même si cette décision aurait eu le mérite de supprimer tout conflit d'intérêt entre la politique monétaire et la surveillance bancaire. Il considérait en effet que l'autorité de la BoE dans la City découlait du fait qu'elle était chargée de la surveillance du système bancaire. Il lui paraissait important qu'elle le restât, et la nouvelle loi lui accorda même davantage de pouvoir concernant les règles prudentielles à faire respecter lors des opérations de prise de contrôle d'un établissement bancaire. La BoE pouvait désormais, au nom de la prudence, s'opposer à toute prise de contrôle d'une banque inscrite au Royaume-Uni, car la prise de participation d'une banque au capital d'une autre banque n'augmente pas la capitalisation globale du système, et par voie de conséquence n'augmente pas la protection contre le risque, même si elle renforce la position de la banque.

La loi de 1987 mettait en place une surveillance des activités bancaires beaucoup plus structurée, même si la BoE avait toujours l'intention d'utiliser les procédures informelles et le dialogue, et de ne recourir aux pouvoirs que lui conférait le législateur que dans les cas graves et une fois que toutes les voies informelles auraient été épuisées. C'est ce qu'on peut déduire du discours du sous-gouverneur de la BoE, Sir George Blunden, prononcé devant la Lombard Association le 8 avril $1987^{44}$.

\section{Conclusion}

La déréglementation financière, qui rendit le système financier britannique beaucoup plus concurrentiel, mit fin à la spécialisation des acteurs britanniques, au cloisonnement des marchés, et à l'existence des «deux Cities». Elle renforça le rôle international de la City au profit des mastodontes américains, japonais et européens, mais au détriment des acteurs britanniques. Avec la loi sur les services financiers de 1986 et la loi bancaire de 1987, la réglementation bancaire et financière au RoyaumeUni prit une forme inconnue jusque-là. La réglementation, qui toucha désormais tous les acteurs et tous les secteurs et devint extrêmement détaillée et normative, mit fin à ce qui subsistait du système d'autorégulation. Si la Banque d'Angleterre paraît avoir été très peu associée à la conception de la loi sur les services financiers, l'accord ParkinsonGoodison montre qu'elle joua un rôle non négligeable dans la déréglementation de la Bourse de Londres, et qu'elle parvint à convaincre le gouvernement, grâce à la prise de position du chancelier de l'Échiquier, qu'elle devait rester le régulateur des banques, alors que ce rôle était très menacé par le pouvoir politique. 


\section{BIBLIOGRAPHIE}

\section{Archives}

\section{Bank of England}

Sir Kenneth Couzens to Geoffrey Howe, Gordon Richardson, Nigel Lawson, Douglas Dawkins and David Walker, 17 September 1979, Bank of England Archives, Exchange Control Department, File EC5 $/ 652$

Sir Geoffrey Howe, Letter to Margaret Thatcher, 11 octobre 1979, Bank of England Archives, Exchange Control Department, File EC5/652.

Gordon Richardson, Letter to Chancellor of the Exchequer, 11 mai 1979, Bank of England Archives, File G3/355.

\section{The National Archives (TNA)}

HM Treasury, Economic Progress Report sent to Margaret Thatcher, Oct.1981, Records of the Prime Minister's Office: Correspondence and Papers, 1979-1997. ECONOMIC POLICY. Exchange rate; exchange control policy ; inflow controls ; part 1.4 mai 1979 - 23 octobre 1981, TNA, PREM $19 / 437$.

Prime Minister's Office, Correspondence and Papers, 1979-1997, Douglas Hague letter to MT ("Exchange Control"), 4 mai 1979, TNA, PREM19/437.

\section{Références}

BELLRINGER Christopher, MICHIE Ranald, «Big Bang in the City of London: an intentional revolution or an accident », Financial History Review, vol. 21, n²,_août 2014, p. 111-137.

BLUNDEN George (Sir), "Supervision and Central Banking », Speech by the Deputy Governor to the Lombard Association, 8 avril 1987, Bank of England Quarterly Bulletin, vol. 27, n³, 3 août 1987, p. 380-386.

воотн Philip, « Thatcher: the Myth of Deregulation », IEA Discussion Paper No. 60, mai 2015, <https://iea.org.uk/publications/research/thatcher-the-myth-of-deregulation> CAPIE Forrest, The Bank of England: 1950s to 1979, Cambridge, Cambridge University Press, 2010, 920 p.

CASSIS Youssef, Les capitales du capital, 1780-2005, Genève, Editions Slatkine, 2006, 453 p.

CONSERVATIVE PARTY, The Right Approach to the Economy, Londres, 1977, <https:// www.margaretthatcher.org/document/112551> CONSERVATIVE PARTY, 1979 Conservative Manifesto, <www.conservative-party.net/manifestos/1979/ 1979-conservative-manifesto.shtml> 
ESPOSITO Marie-Claude, La politique industrielle des gouvernements conservateurs d'Edward Heath et de Margaret Thatcher, Thèse de Doctorat d'État de l'Université de Paris Sorbonne, dirigée par le professeur André Guillaume, janvier 1990, tome 1.

ESPOSITO Marie-Claude « La déréglementation financière au Royaume-Uni : un peu d'histoire » in AZUELos Martine (dir.), La déréglementation des économies anglo-saxonnes, Paris, PSN, 1995, p. 89-97.

ESPOSITO Marie-Claude, «L'irrésistible ascension de la place financière de Londres depuis le milieu des années 1950 », in KORINMAN Michel (dir.), L'avenir économique du monde, Économie et géopolitique. 1, Bègles, Collection Outre-Terre, 2016, p. 106-130.

HOUSE OF COMMONS, Hansard, 12 juin 1979, vol. 968, col. 235-263.

HOWE Geoffrey, Conflict of Loyalty, New York, St. Martin's Press, 1994, 736 p.

LAWSON Nigel, The View from Number 11, Londres, Bantam Press, 1992, 1152 p.

LERUEZ Jacques, Le Phénomène Thatcher, Bruxelles, éditions Complexe, 1991.

LERUEZ Jacques, Thatcher, la dame de fer, Bruxelles, éditions André Versaille, 2012.

MICHIE Ranald et WILLIAMSON Philip (dir.), The British Government and the City of London in the 20th Century, Cambridge, Cambridge University Press, 2004, 381 p.

MICHIE Ranald, «A Financial Phoenix : The City of London in the Twentieth Century ", in CASSIS Youssef et BUISSIÈRE Éric (dir.), London and Paris as International Financial Centres in the Twentieth Century, Oxford, Oxford University Press, 2005, p. 15-41.

SCHENK Catherine R., « The Origins of the Eurodollar Market in London, 1953-1963 », Explorations in Economic History, vol. 35, n², 1998, p. 221-238.

SCHENK Catherine, « Crisis and Opportunity: The Policy Environment of International Banking in the City of London, 1958-1980 », in CASSIS Youssef et BUISSIÈre Éric (dir.), London and Paris as International Financial Centres in the Twentieth Century, Oxford, Oxford University Press, 2005, p. 207-228.

ROBERTS Richard, « The Bank and the City », in ROBERTS Richard and KYNASTON David (dir.), The Bank of England: Money, Power and Influence, 1694-1994, Oxford, Oxford University Press, 1995, 315 p.

THATCHER Margaret, The Downing Street Years, Londres, HarperCollins, 1993, 914 p.

\section{NOTES}

1. Pour plus de détails, on pourra se reporter très utilement à deux ouvrages de Jacques Leruez : Le Phénomène Thatcher, Bruxelles, éditions Complexe, 1991; Thatcher, la dame de fer, Bruxelles, éditions André Versaille, 2012.

2. воотн Philip, "Thatcher: the Myth of Deregulation », IEA Discussion Paper No. 60, mai 2015, https://iea.org.uk/publications/research/thatcher-the-myth-of-deregulation.

3. Voir également ESPOSITO Marie-Claude, «La déréglementation financière au Royaume-Uni : un peu d'histoire » in AzUelos Martine (dir.), La déréglementation des économies anglo-saxonnes, Paris, PSN, 1995, p. 90-91, et ESPOSITO Marie-Claude, «L'irrésistible ascension de la place financière de Londres depuis le milieu des années 1950 », in KoRINMAN Michel (dir.), L'avenir économique du monde, Économie et géopolitique 1, Bègles, Collection Outre-Terre, 2016, p. 108-111. 
4. Ceux-ci étaient placés à Londres par les banques américaines souhaitant bénéficier de taux plus rémunérateurs, la FED ayant imposé la réglementation Q qui plafonnait la rémunération des dépôts en dollars sur le territoire américain.

5. SCHENK Catherine R., "The Origins of the Eurodollar Market in London, 1953-1963", Explorations in Economic History, vol. 35, n², 1998, p. 224-226.

6. Il passa de 82 en 1961 à 159 en 1970. Voir MICHIE Ranald, «A Financial Phoenix: The City of London in the Twentieth Century ", in CASSIS Youssef et BuISSIÈRE Éric (dir.), London and Paris as International Financial Centres in the Twentieth Century, Oxford, Oxford University Press, 2005, p. 31.

7. Le fonctionnement du système international de Bretton Woods les contraignait à racheter les dollars du déficit américain qu'elles venaient placer sur ce marché.

8. CASSIS Youssef, Les capitales du capital, 1780-2005, Genève, Editions Slatkine, 2006, p. 312.

9. scHENK Catherine, «Crisis and Opportunity: The Policy Environment of International Banking in the City of London, 1958-1980 », in CASSIS Youssef et BUISSIÈre Éric (dir.), London and Paris as International Financial Centres in the Twentieth Century, op. cit., p. 212.

10. SCHENK Catherine, «Crisis and Opportunity: The Policy Environment of International Banking in the City of London, 1958-1980 », op. cit., p. 237.

11. MICHIE Ranald et WILLIAMson Philip (dir.), The British Government and the City of London in the Twentieth Century, Cambridge, Cambridge University Press, 2004, p. 44.

12. Il comprenait un accord entre les banques sur un taux minimum pour les prêts au jour le jour aux maisons d'escompte, un prix d'offre unique proposé par les maisons d'escompte à l'adjudication hebdomadaire des bons du Trésor, et l'engagement de la BoE que les maisons d'escompte puissent se refinancer selon la pratique du prêt en dernier ressort. Voir ESPOSITO Marie-Claude, La politique industrielle des gouvernements conservateurs d'Edward Heath et de Margaret Thatcher, Thèse de Doctorat d'État de l'Université de Paris Sorbonne, dirigée par le professeur André Guillaume, janvier 1990, tome 1, p. 82.

13. LAWSON Nigel, The View from Number 11, Londres, Bantam Press, 1992, p. 39.

14. HowE Geoffrey, Conflict of Loyalty, New York, St. Martin's Press, 1994, p. 141.

15. CONSERVATIVE PARTY, The Right Approach to the Economy, Londres, 1977.

16. CONSERVATIVE PARTY, 1979 Conservative Manifesto, www.conservative-party.net/manifestos/ 1979/1979-conservative-manifesto.shtml.

17. Il sera chancelier de l'Échiquier de 1983 à 1989.

18. Nigel Lawson, The View from Number 11, op. cit., p. 38.

19. Douglas Hague's letter to MT ("Exchange Control"), 4 mai 1979, TNA, PREM 19/437.

20. Gordon Richardson's letter to Chancellor of the Exchequer, 11 mai 1979, Bank of England Archives, File G3/355.

21. Sir HOWE Geoffrey, Letter to Margaret Thatcher, 11 octobre 1979, Bank of England Archives, File EC5/652.

22. Voir la note du 17 septembre 1979 envoyée par Sir Kenneth Couzens à Geoffrey Howe, Gordon Richardson, Nigel Lawson, Douglas Dawkins et David Walker. Bank of England Archives, Exchange Control Department, File EC5/652.

23. D'après Howe, Margaret Thatcher a très facilement accepté la première étape du processus, mais difficilement les mesures prises à l'automne. Voir HOWE Geoffrey, Conflict of Loyalty, op. cit., p. 142. Quant à Margaret Thatcher, elle ne mentionne à aucun moment la question du contrôle des changes dans ses mémoires. Voir THATCHER Margaret, The Downing Street Years, Londres, HarperCollins, 1993.

24. Les estimations du Treasury concernant le montant des fuites de capitaux furent très en deçà de la réalité. Ex post, les flux sortants atteignirent 4 milliards de livres à partir de 1981, soit deux fois plus que les prévisions du Treasury. Voir HM Treasury, Economic Progress Report sent to Margaret Thatcher, Oct. 1981, Records of the Prime Minister's Office: Correspondence and 
Papers, 1979-1997. ECONOMIC POLICY. Exchange rate; exchange control policy ; inflow controls ; part 1. 4 mai 1979 - 23 octobre 1981. TNA, PREM 19/437.

25. LAWSON Nigel, The View from Number 11, op. cit., p. 39.

26. Pour le détail des mesures, voir également EsPosito Marie-Claude, La politique industrielle des gouvernements conservateurs d'Edward Heath et de Margaret Thatcher, op. cit., p. 316.

27. HOUSE OF COMMONS, Hansard, 12 juin 1979, vol. 968, col. 235-263.

28. LAWSON Nigel, The View from Number 11, op. cit., p. 40.

29. HOWE Geoffrey, Conflict of Loyalty, op. cit., p. 142.

30. Ces suppressions d'emplois furent gérées grâce à des pré-retraites, au redéploiement de postes au sein de la BoE, et à l'acceptation d'emplois dans la City. Voir LAwson Nigel, The View from Number 11, op. cit., p. 40, et CAPIE Forrest, The Bank of England: 1950s to 1979, Cambridge, Cambridge University Press, 2010, p. 770.

31. HMT, Economic Progress Report sent to Margaret Thatcher, Oct 1981, Records of the Prime Minister's Office: Correspondence and Papers, 1979-1997, op. cit.

32. BELLRINGER Christopher et MICHIE Ranald, «Big Bang in the City of London: an intentional revolution or an accident ", Financial History Review, vol. 21, n², août 2014, p. 122.

33. Pour plus de détails, voir ESPOSITO, M-C., La politique industrielle des gouvernements conservateurs d'Edward Heath et de Margaret Thatcher, op. cit., p. 79-85.

34. ESPOSITO Marie-Claude, «La déréglementation financière au Royaume-Uni », op. cit., p. 93.

35. Une analyse détaillée, à partir des archives disponibles, est donnée dans BELLRINGER Christopher et MICHIE Ranald, «Big Bang in the City of London: an intentional revolution or an accident? », op. cit, p. 124-126.

36. Elle empêchait tout conflit d'intérêt entre les jobbers (les contrepartistes) et les brokers (agents de change) qui transmettaient les ordres de la clientèle aux jobbers.

37. Il s'agit du SEAQ (Stock Exchange Automated Quotation).

38. Teletext Output of Price Information by Computer.

39. Voir EsPosito Marie-Claude, «L'irrésistible ascension de la place financière de Londres depuis le milieu des années 1950 », op. cit., p. 114-115.

40. Cette théorie, qui découle des travaux de George Stigler, décrit une situation où une institution publique de régulation, qui se doit d'agir en faveur de la collectivité, finit par servir les intérêts privés.

41. LAWSON Nigel, op. cit., p. 401.

42. LAWSON Nigel, op. cit., p. 403-405.

43. Ibidem, p. 408.

44. Sir BLUNDEN George, "Supervision and Central Banking ", Speech by the Deputy Governor to the Lombard Association, 8 avril 1987, Bank of England Quarterly Bulletin, vol. 27, n³, 3 août 1987, p. 380-386.

\section{RÉSUMÉS}

La politique économique de Margaret Thatcher de 1979 à 1990 s'est voulue en rupture avec les politiques macro-économiques keynésiennes mises en œuvre depuis la fin de la Seconde Guerre mondiale. Elle eut pour premier objectif la lutte contre l'inflation, et pour second objectif la réduction du rôle de l'état dans l'économie pour améliorer les performances de l'offre globale, 
dont dépendait la croissance, ce qui impliquait d'une part de déréglementer les marchés, et d'autre part de privatiser des pans entiers du secteur public. Cet article se limite à l'étude de la déréglementation des marchés financiers dont la contribution en termes de production, d'emploi et d'exportations de services affichait une progression régulière depuis le début des années 1970. Les forces et les faiblesses de la City à la fin des années 1970 sont présentées dans une première partie. Les principales mesures de déréglementation des marchés financiers font l'objet de la deuxième partie. La troisième partie explique pourquoi le gouvernement a élargi le champ de la réglementation financière et durci la réglementation bancaire.

Margaret Thatcher's economic policy from 1979 to 1990 was meant to break away from the economic practices of successive post-war Keynesian governments. Fighting inflation was its first objective and reducing the government's presence in the economy to improve its supply-side, and growth in particular, its second objective. As a result, markets were deregulated and stateowned industries were privatized. This article deals with the deregulation of financial markets whose contribution in terms of wealth, job creation and exports had been rising since the beginning of the 1970s. The strengths and weaknesses of the City of London are analysed in a first part and the deregulation of financial markets in a second part. The third section explains why the government introduced regulation into areas which had never been subject to statutory regulation, and why it broadened the scope of financial regulation and strengthened banking supervision.

\section{INDEX}

Mots-clés : Banque d'Angleterre, Big Bang, City, contrôle des changes, Securities Investment Board (SIB), Treasury

Keywords : Bank of England, Big Bang, City, exchange controls, Securities Investment Board, Treasury

\section{AUTEUR}

\section{MARIE-CLAUDE ESPOSITO}

Professeur émérite, Sorbonne Nouvelle, EA 2291, ICEE-Intégration et coopération dans l'espace européen 